\title{
Lymph nodes morphology as predictor natural and premature aging
}

\author{
Olga Gorchakova \\ Research Institute of a clinical and \\ experimental Lymphology - branch of \\ Institute of Cytology and Genetics of the \\ Siberian Branch of the Russian Academy \\ of Science \\ Novosibirsk, Russia \\ ORCID 0000-0001-7732-7587
}

\author{
Vladimir Gorchakov \\ Research Institute of a clinical and \\ experimental Lymphology - branch of \\ Institute of Cytology and Genetics of the \\ Siberian Branch of the Russian Academy \\ of Science, \\ Novosibirsk State Universite \\ Novosibirsk, Russia \\ ORCID 0000-0001-8135-7842 \\ vgorchak@yandex.ru
}

\author{
Georgy Demchenko \\ Institute of Physiology of Human and \\ Animals of Committee of Science of the \\ Ministry of Education and Science of the \\ Republic of Kazakhstan \\ Almaty, Kazakhstan \\ ORCID 0000-0001-9906-2700
}

\begin{abstract}
The research purpose is to estimate the structural organization of lymph nodes of different localization at natural (physiological) and premature aging respectively at OXYS and Wistar rats. We used a morphological method of a research. The age-induced changes of lymphoid tissue of Wistar and OXYS rats differ with type of the immune response and morphological variant of lymph nodes structure. The immune response on humoral type is formed in mesenteric and inguinal lymph nodes, and the immune response on cellular type is formed in a tracheobronchial lymph node. We noted reduction of structures of cortical substance, especially lymphoid follicles and a paracortex, and expansion of medullary substance in a lymph node of OXYS rats. Lymph nodes are subject big morphological changes at rats of OXYS, than at old rats of Wistar. Observed changes of structure of lymph nodes of OXYS rats are a morphological equivalent of premature aging and confirms early decrease of a drainage and immune function of lymph nodes.
\end{abstract}

Keywords - lymph node, OXYS and Wistar rats, premature aging, gerontology

\section{Introduction}

Clarification of the reasons of aging of lymphoid tissue has special relevance because of increase in number of old people in the country and need of specification of pathogenesis and search of method of prevention. Lymph nodes are key elements of different lymphatic regions, and lymph nodes define a regional immunological homeostasis [1,2]. There is an urgent need of a morph of functional assessment of lymph nodes depending on localization taking into account an age factor. There is no uniform theory of aging [3]. Researchers combine often the mechanism of aging and pathogenesis of immune insufficiency. It is impossible to consider separately age evolution and aging of lymphoid tissue without structural reorganization of lymph nodes. Emergence of model of senilism of the line of rats of OXYS [4] is an argument for studying morphological equivalents of aging of lymph nodes. Morphology of lymph nodes at OXYS rats are not provided..

The purpose of work is a research of age changes of lymph nodes of different localization at OXYS rats with a syndrome of premature aging and Wistar rats with the normal rate of aging.

\section{Material and methods}

Work is performed on rats males of the OXYS and Wistar lines according to "Rules of work with use of experimental animals" (86/609/EEC). Rats of the Wistar and OXYS lines are received from Center of collective use "Gene pools of laboratory animals" of Institute of cytology and genetics of the SB RAS. N.G. Kolosova is a holder of the license for OXYS rats [4]. These rats have genetically caused defect, it is considered as an accelerated aging syndrome.

The experiment was executed on young and old white rats. The age of rats is 3 months and 1.5 years. We investigated by a morphological method of mesenteric, inguinal and tracheobronchial lymph nodes. Lymph nodes fixed in $10 \%$ neutral formalin. After fixing we adhered to the classical scheme of washing, dehydration, imbibition with a xylol, paraffin and preparation histologic sections on the microtome. Histologic sections of lymph nodes painted hematoxylin and eosine, azureII-eosine, trichromatic paint on Masson.

The morphometric analysis of structures of a lymph node was carried out by means of a morphometric grid and the ImagePro Plus 4.1 program. Statistical data processing was performed with licensed statistical software package StatPlus Pro 2009, AnalystSoff Inc. A P-value $<0.05$ was considered statistically significant.

\section{Results and Discussion}

At natural (physiological) aging of involution of lymphoid tissue the stage of the maximum development of compartments of a lymph node precedes that corresponds three-month-old of young rats of Wistar $[1,5]$. The indicator of a ratio of T- and Bzones makes more than unit at three months age of young rats, building a progressive row of lymph nodes: tracheobronchial inguinal - mesenteric. Each of these lymph nodes differs with extent of development of structural and functional zones. Involution of adenoid tissue leads to structural destabilization of regional lymph nodes at old rats of Wistar.

The coefficient of a ratio of $\mathrm{T}$ - and B-zones almost changes in an inguinal lymph node at natural aging. The coefficient considerably decreases in a mesenteric lymph node and increases in a tracheobronchial lymph node. So, immunoactive zones have the following changes by 1.5 years at rats of Wistar.

The inguinal lymph node. There is an expansion of the area of the cortical plateau (in 1.2 times) a medullary sine and medullary cords (in 1.4-1.5 times) and reduction of the area of paracortical area (in 1.2 times), lymphoid follicles (in 1.9 times) in the inguinal lymph node.

The mesenteric lymph node. There is a reduction of the area of the cortical plateau (in 1.9 times), a paracortex (in 1.8 times), lymphoid follicles with the germinative center (in 4 times), a 
medullary sine (in 1.5 times) and expansion medullary cords (in 1.8 times) in the mesenteric lymph node.

The tracheobronchial lymph node. There is a reduction by 1.2-1.3 times of the area of the cortical plateau (in 1.2 times), a paracortex (in 1.3 times), lymphoid follicles (in 4.5 times), medullary cords (in 1.2 times) with an invariable width of a medullary sine in the tracheobronchial lymph node.

It is clear, there is a redistribution of the immunoactive compartments which are responsible for the immune response in lymph nodes of different lymphatic regions with age.

Other morphological picture is observed in lymph nodes of premature aging of OXYS rats. Changes of lymphoid tissue are characterized more rapidly development without achievement of the maximum development of compartments. The greatest changes are noted $\mathrm{T}$ - and B-zones of lymph nodes. These zones are responsible for forming of the immune response on cellular and humoral type. The coefficient of a ratio of $\mathrm{T}$ - and $\mathrm{B}$-zones less than unit also demonstrates prevalence in structure of lymph nodes a thymus-independent area at of OXYS rats at threemonths age. Indicators of structural and functional zones of lymph nodes are various at three-months age of OXYS and Wistar rats. We noted the following changes in lymph nodes of young rats of OXYS.

The inguinal lymph node. There is an increase in the area of the cortical plateau (in 1.9 times), a medullary sine (in 1.2 times) and reduction of the area of a paracortex (in 1.4 times), lymphoid follicles (in 3.4 times), medullary cords (in 1.6 times) in an inguinal lymph node

The mesenteric lymph node. There is a reduction of the sizes of the cortical plateau (in 1.8 times), a paracortex (in 2.8 times), lymphoid follicles (in 3.3 times), and increase in a medullary sine (in 1.5 times) in a mesenteric lymph node.

The tracheobronchial lymph node. There is a reduction of the area occupied by the cortical plateau (in 1.2 times), a paracortex (in 1.3 times), lymphoid follicles (in 3.4 times) and increase in the area occupied by medullary cords (in 1.3 times) at minor change of a medullary sine in a tracheobronchial lymph node.

The coefficient of a ratio of T- and B-zones remains low size in inguinal and mesenteric lymph nodes and increases in a tracheobronchial lymph node when aging by 1.5 years. It is connected with regress of the main structural and functional zones (a paracortex, lymphoid follicles) at expansion of medullary substance in lymph nodes of different lymphatic regions. Nevertheless, the T-zone dominates in a tracheobronchial lymph node that defines the immune response of cellular type. Changes of the structural organization of lymph nodes are the morphological sign of decrease immune protection progressing in process of aging at OXYS rats.

The structure of lymph nodes of OXYS old rats does not provide a drainage and the immune status of the lymphatic region. It increases risk of developing pathology [3]. It should be considered by search of means of correction of senile changes of lymphoid tissue for increase of nonspecific resistance [5].

\section{Conclusion}

Morphodynamics of compartments of lymph nodes of different localization has distinctions at natural and premature aging. The imbalance of a ratio of T- and B-zones characterizes depression of function of lymph nodes depending on localization and age. The general is minimization of structures of cortical substance and increase medullary substance of lymph nodes. Changes of compartments sizes lead to forming of the immune response of humoral type for a mesenteric and inguinal lymph nodes and the immune response of cellular type for a tracheobronchial lymph node. At the same time at rats of OXYS the aging happens the accelerated rates and regressive changes of lymphoid tissue are available already for young animals. The age-caused imbalance of compartments of a lymph node can be considered as a predictor of premature aging and as risk of developing pathology. Features of morphology of lymph nodes give the grounds to consider of OXYS rats as universal model of immune insufficiency for studying pathogenesis and development of correction methods.

\section{ACKNOWLEDGMENT}

The state task to research work \# 0324-2019-0045-C-02, AAAA-A19-119031590017-7.

\section{REFERENCES}

[1] Ju.I. Borodin, O.V. Gorchakova, A.V. Suhovershin and V.N. Gorchakov The concept of lymphatic region in preventive lymphology. LAP LAMBERT Academic Publishing, 2018, 74 p.

[2] H. Suami Lymphosome concept: Anatomical study of the lymphatic system / H. Suami // Journal of Surgical Oncology, 2017, vol. 115, no 1, pp.1-5. DOI: 10.1002/jso.24332.

[3] E.G. Zotkin, I.S. Dydykin, Lila A.M. The inflammatory theory of aging, age-associated diseases and osteoarthritis // Russian medical journal, 2020, no. 7, pp.33-38.

[4] N.G. Kolosova, N.A. Stefanova, E.E. Korbolina, A.Zh. Fursova and O.S. Kozhevnikova Senescence-Accelerated OXYS Rats: A Genetic Model of Premature Aging and Age-Related Diseases // ADVANCES IN GERONTOLOGY, 2014, vol. 27, no. 2, pp. 336-340.

[5] O.V. Gorchakova and V.N. Gorchakov, Increase in drainage and immune functions of a lymph node as a factor of endoecological wellbeing at advanced and senile age // ADVANCES IN GERONTOLOGY, 2015, vol. 28, no. 3, pp. 521-526. 